\title{
COUPLED THERMAL AND MECHANICAL ANALYSIS OF COMPOSITE CROSS SECTIONS USING MATHEMATICAL OPTIMIZATION STRATEGIES
}

\author{
Christopher TAUBE, Hans-Georg TIMMLER, Marcel HELMRICH, Guido MORGENTHAL \\ Department Simulation and Modelling of Structures, Faculty of Civil Engineering, Bauhaus-Universität \\ Weimar, Marienstraße 13a, 99421 Weimar, Germany
}

Received 29 August 2016; accepted 30 September 2016

\begin{abstract}
In the present article an alternative approach for the coupled thermal and mechanical analysis of composite cross sections under temperature effects is introduced, which uses the mathematical optimization as a consistent methodical base. By applying the principle of the virtual source energy for the thermal and the principle of the minimum of the total potential energy for the mechanical analysis, an accurate determination of temperature fields as well as residual strain and stress distributions is possible. The coupling is enabled by the thermal strains, which are determined based on the temperature field and passed to the nonlinear mechanical analysis as tension free pre-strains. The energy functional of the heat conduction problem is derived and implemented. The resulting optimization task is strictly convex and represents an implicit formulation, which does not impose any stability criteria. The performance of the introduced method is demonstrated on a principle example and an outlook is given on possible further extensions and applications.
\end{abstract}

Keywords: composite cross sections, thermal effects, restraint effects, heat conduction, energy principles, variation principles, optimization task, transient thermal analysis, nonlinear mechanical analysis.

\section{Introduction}

Thermal effects lead to mechanical deformations of structures. When such deformations are restrained, they create internal stresses through the coupling of thermal and mechanical behaviour. Such restraint effects are of importance to many structures, for example in relation to environmental effects (e.g. solar radiation), to fire conditions or under the influence of internal energy sources such as hydration processes in concrete. Purely mechanical restraint effects are possible due to imposed deformations such as creep and shrinkage of concrete or differential support settlement of structures.

Restraint effects may be significant to the design of structures, e.g. in integral bridges (Jung et al. 2013; Morgenthal, Olney 2016) and their accurate prediction therefore requires appropriate simulation techniques which combine the thermal and mechanical analysis and the accurate consideration of influencing parameters. A framework for such analysis is presented in this paper.

Figure 1 gives an overview on the approach for the determination of temperature fields and residual strain distributions in composite cross sections subjected to thermal constraints. For the temperature field analysis, thermal material properties like the thermal conductivity $\lambda$, the density $\rho$ and the heat capacity $c$, which may be temperature and/or time dependent, have to be considered. Furthermore, thermal boundary and matching conditions, like given temperature functions or heat flows at the edges or heat transition between two materials, have to be satisfied. The coupling is enabled by the thermal strains, which are computed based on the determined temperature field and passed to the

Corresponding author:

Ch. Taube E-mail: christopher.taube@uni-weimar.de 
mechanical analysis as tension free pre-strains. The residual strains $\varepsilon_{\sigma}$ are the result of the mechanical analysis, which is performed under consideration of the mechanical material properties (which can be temperature and/or time dependent) and under satisfaction of the compatibility conditions for the total strains $\varepsilon_{t o t}$, like e. g. the Bernoulli-hypothesis, and static boundary conditions. The scheme shows, that the results of the mechanical analysis are highly sensitive to uncertainties in the calculation of the temperature field, because of the error in the committed thermal strain distribution as well as the high temperature dependency of the mechanical material parameters, especially in the cases of fire conditions or young concrete (Achenbach, Morgenthal 2014). Thus, an accurate thermal analysis is strictly necessary.

Closed analytical solutions do only exist for special cases of the heat conduction problem. Usually, numerical approximations based on the Finite Element Method find a use (Baehr, Stephan 2013; Häfner et al. 1992). An alternative approach for the calculation of temperature fields in composite cross sections is introduced in the present article. It is based on a direct transformation of the extremum principle of heat conduction into an optimization task, which can be solved using solver-tools for nonlinear optimization, which are implemented in mathematical standard software like MS Excel or Matlab.

The mechanical analysis will be also realized using an alternative method, which is based on the minimum of the potential energy by Lagrange and on a kinematical description of the mechanical problem. The resulting optimization task can be solved by standard software, too. This approach for the mechanical analysis of composite cross sections under load and/or constraint influences is well investigated and published (Raue 2005, 2007; Schröter 2014).

By using these alternative methods, combined thermal and mechanical phenomena in composite cross sections under arbitrary combinations of loads and temperature constraints can be depicted. Due to the degree of nonlinearity, fire exposed and young concrete are taking on special positions, because of the temperature and time dependency of the thermal and mechanical material properties. In the present article, a fire exposed cross section will be taken as a principle example for illustrating the performance of the introduced method.

\section{Thermal analysis of composite cross sections}

\subsection{The functional form of the heat conduction problem}

For showing the basics of the calculation method, a domain $G$ which is exposed to thermal influences within the time interval $T=\left[t_{1}, t_{2}\right]$ is considered as shown in Figure 2. The edge $R$ of the domain is subdivided into 3 parts, that represent different boundary conditions: fixed temperature function $f\left(R_{1}\right)$ and fixed flux density function $q_{n}\left(R_{2}\right)$ in normal directions as well as linear combinations of both of them $\left(R_{3}\right)$ to satisfy transition conditions between adjoining materials. Further, the domain is influenced by an internal heat source $W$,

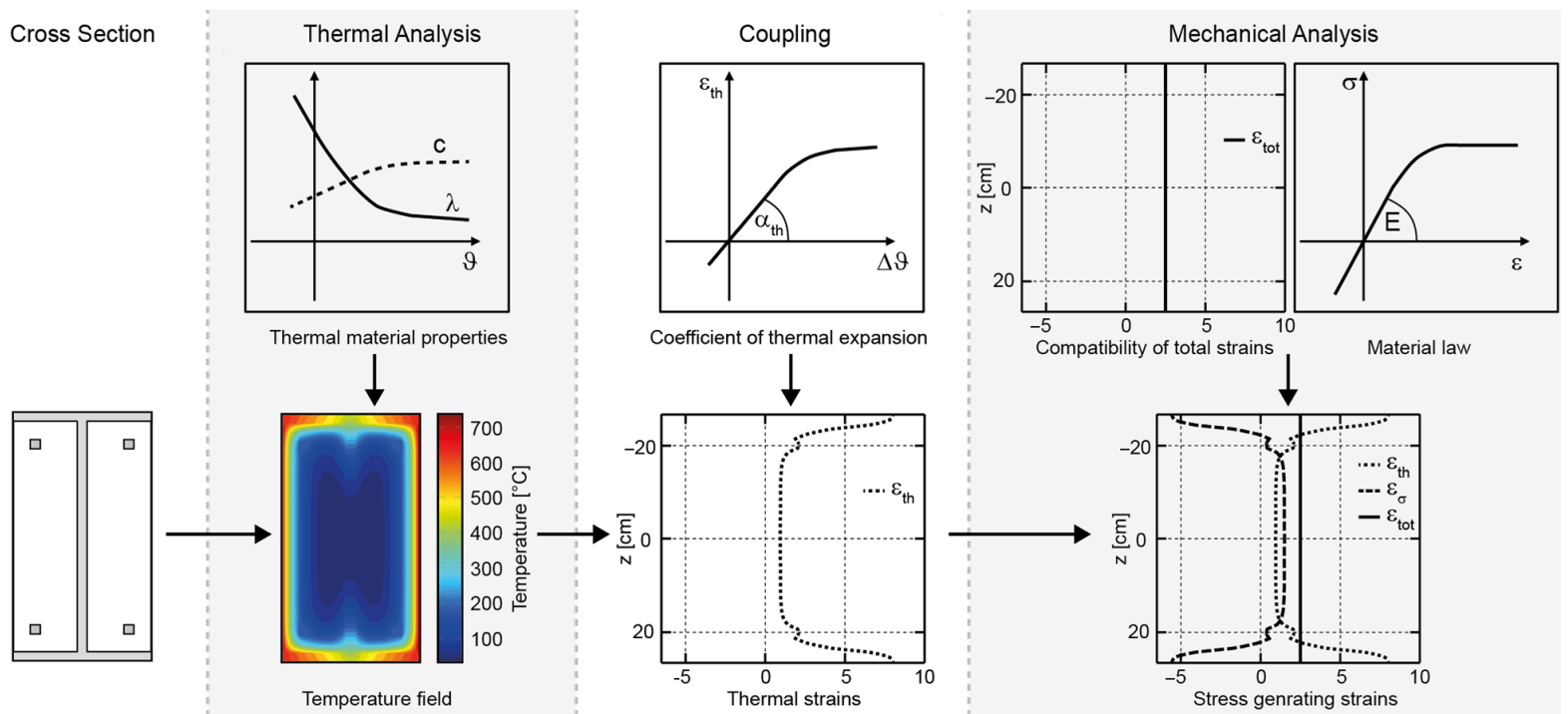

Fig. 1. Scheme for the approach of coupling the mechanical analysis with an upstream thermal analysis 


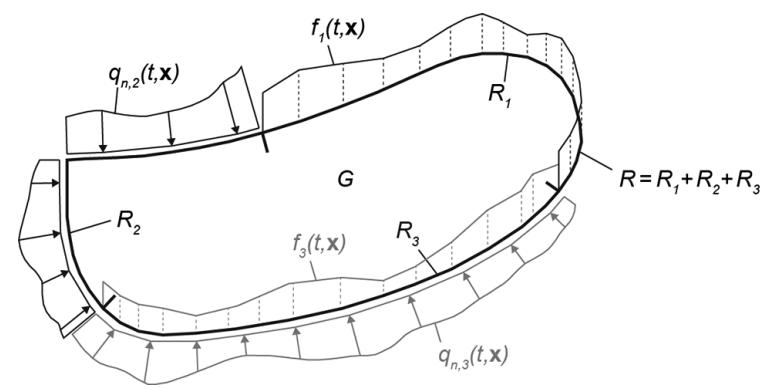

Fig. 2. Domain $G$ and edge $R$ of the heat conduction problem (Kaempf 1983)

which has a temperature, place and time dependency. The material properties are assumed to be temperature dependent.

Analogous to other physical field problems, the heat conduction problem can be expressed as an extremum principle. From all possible temperature distributions inside the cross section, the one will occur, for which the total energy reaches a minimum. The corresponding functional equation can be derived from the partial differential equation of heat conduction:

$$
\rho(\vartheta) c(\vartheta) \frac{\partial \vartheta}{\partial t}=\nabla^{\mathrm{T}}[\lambda(\vartheta) \nabla \vartheta]+W(\vartheta, x, t),
$$

where: $\vartheta$ - temperature; $\rho$ - density; $c$ - specific heat capacity; $\lambda$ - thermal conductivity; $W$ - internal heat sources; $t$-time; $\nabla$ - Nabla-operator.

For making the derivation more clear, the following conventions will be made:

$$
\begin{aligned}
& c(\vartheta)=c, \quad \rho(\vartheta)=\rho, \quad \lambda(\vartheta)=\lambda, \quad \kappa=\frac{1}{c \rho}, \\
& \frac{\partial()}{\partial t}=(\dot{)}), \frac{\partial()}{\partial \vartheta}=()^{\prime} .
\end{aligned}
$$

Considering this conventions, Eq. (1) can be expressed as

$$
\dot{\vartheta}-\kappa \nabla^{\mathrm{T}}[\lambda \nabla \vartheta]-\kappa W=0 .
$$

The multiplication of Eq. (3) by a virtual variation of the specific thermal source energy $\delta e$, leads to

$$
\iint_{T G}\left(\dot{\vartheta}-\kappa \nabla^{\mathrm{T}}[\lambda \nabla \vartheta]-\kappa W\right) \delta e \mathrm{~d} V \mathrm{~d} t=0 .
$$

By setting this specific thermal source energy to

$$
e=\dot{\vartheta}-\kappa \nabla^{\mathrm{T}}[\lambda \nabla \vartheta]
$$

the insertion into Eq. (4) results in the following statement:

$$
\iint_{T G}(e-\kappa W) \delta e \mathrm{~d} V \mathrm{~d} t=0
$$

This expression is denoted as the principle of the virtual source energy, which is a special form of the virtual energy transmission (Mazilu 1982; Kaempf 1983). The Transformation to

$$
\delta \int_{T} \int_{G}\left(\frac{1}{2} e^{2}-\kappa W e\right) \mathrm{d} V \mathrm{~d} t=0,
$$

enables the exposition in the substituted and resubstituted functional form:

$$
\begin{gathered}
\Phi=\int_{T G} \int_{G}\left(\frac{1}{2} e^{2}-\kappa W e\right) \mathrm{d} V \mathrm{~d} t \\
\Phi=\int_{T G} \int_{G}\left(\begin{array}{l}
\frac{1}{2}\left(\dot{\vartheta}-\kappa \nabla^{\mathrm{T}}[\lambda \nabla \vartheta]\right)^{2}- \\
\kappa W\left(\dot{\vartheta}-\kappa \nabla^{\mathrm{T}}[\lambda \nabla \vartheta]\right)
\end{array}\right) \mathrm{d} V \mathrm{~d} t .
\end{gathered}
$$

The numerical evaluation of this expression is relatively difficult. Thus, it has to be transformed for better implementation into program codes. The expansion of the functional terms in Eq. (9) leads to:

$$
\begin{aligned}
& \Phi=\frac{1}{2} \int_{T G} \int_{G} \dot{\vartheta}^{2} \mathrm{~d} V \mathrm{~d} t-\int_{T G} \int_{G} \kappa \nabla^{\mathrm{T}}[\lambda \nabla \vartheta] \mathrm{d} V \mathrm{~d} t+ \\
& \frac{1}{2} \int_{T} \int_{G}\left(\kappa \nabla^{\mathrm{T}}[\lambda \nabla \vartheta]\right)^{2} \mathrm{~d} V \mathrm{~d} t- \\
& \int_{T} \int_{G} \kappa W \dot{\vartheta} \mathrm{d} V \mathrm{~d} t+\iint_{T G} \vartheta \kappa^{2} W \nabla^{\mathrm{T}}[\lambda \nabla \vartheta] \mathrm{d} V \mathrm{~d} t .
\end{aligned}
$$

By using the mathematical relation

$$
\begin{aligned}
& \nabla^{\mathrm{T}}\left[\dot{\vartheta}_{\kappa} \lambda \nabla \vartheta\right]= \\
& \nabla^{\mathrm{T}}\left[\dot{\vartheta}_{\kappa}\right] \lambda \nabla \vartheta+\dot{\vartheta}_{\kappa} \nabla^{\mathrm{T}}[\lambda \nabla \vartheta]= \\
& \left(\nabla^{\mathrm{T}} \dot{\vartheta}_{\kappa}+\dot{\vartheta}_{\kappa} \nabla^{\mathrm{T}} \kappa\right) \lambda \nabla \vartheta+\dot{\vartheta}_{\kappa} \nabla^{\mathrm{T}}[\lambda \nabla \vartheta],
\end{aligned}
$$

the second term of Eq. (10) can be transformed into

$$
\begin{aligned}
& \int_{T} \int_{G} \dot{\vartheta}_{\kappa} \nabla^{\mathrm{T}}[\lambda \nabla \vartheta] \mathrm{d} V \mathrm{~d} t=\iint_{T} \nabla^{\mathrm{T}}\left[\dot{\vartheta}_{\kappa} \lambda \nabla \vartheta\right] \mathrm{d} V \mathrm{~d} t- \\
& \int_{T} \int_{G} \nabla^{\mathrm{T}} \dot{\vartheta} \kappa \lambda \nabla \vartheta \mathrm{d} V \mathrm{~d} t-\int_{T} \int_{G} \dot{\vartheta} \nabla^{\mathrm{T}} \kappa \lambda \nabla \vartheta \mathrm{d} V \mathrm{~d} t,
\end{aligned}
$$

where the gradient of the material parameter $\kappa$ is:

$$
\nabla^{\mathrm{T}} \kappa=\nabla^{\mathrm{T}}\left[\frac{1}{c \rho}\right]=-\frac{\nabla^{\mathrm{T}} c \rho+c \nabla^{\mathrm{T}} \rho}{(c \rho)^{2}} .
$$

The gradient used in Eq. (13) cannot be evaluated easily. A simple transformation, as exemplarily shown on the specific heat capacity $c$ below, is able to remedy. For the other material parameters, this transformation can be done analogously:

$$
\nabla^{\mathrm{T}} c=c^{\prime} \nabla^{\mathrm{T}} \vartheta
$$


Thus, Eq. (12) can be written as

$$
\nabla^{\mathrm{T}} \kappa=\kappa^{\prime} \nabla^{\mathrm{T}} \vartheta
$$

where:

$$
\kappa^{\prime}=-\frac{c^{\prime} \rho+c \rho^{\prime}}{(c \rho)^{2}} .
$$

One advantage of the introduced approach is the possibility to consider thermal boundary conditions directly by the evaluation of corresponding terms. Therefore, the first integral term of Eq. (12) can be transformed into a boundary integral by partial integration:

$$
\iint_{T} \nabla^{\mathrm{T}}[\dot{\vartheta} \kappa \lambda \nabla \vartheta] \mathrm{d} V \mathrm{~d} t=\int_{T} \int_{R} \dot{\vartheta} \kappa \lambda \nabla^{\mathrm{T}} \vartheta \mathbf{n} \mathrm{d} A \mathrm{~d} t,
$$

where: $\mathbf{n}$ - normalized normal vector to define the edge.

Because of the boundary condition at edge $R_{3}$ is depicting a linear combination of the conditions at the edges $R_{1}$ and $R_{2}$, the boundary integral in Eq. (17) can be divided into two independent integral terms. By replacing the derivation of the temperature with respect to time by the function $f$ defining the temperature on $R_{1}$

$$
\dot{\vartheta}=\dot{f}(t, \mathbf{x})
$$

and by replacing the thermal conductivity multiplied with the derivation of the temperature with respect to the direction of the vector $\mathbf{n}$ by the flux density function $q_{n}$ on $R_{2}$

$$
\lambda \frac{\partial \vartheta}{\partial \mathbf{n}}=\lambda \nabla^{\mathrm{T}} \vartheta \mathbf{n}=-q_{n}(t, \mathbf{x})
$$

Eq. (17) can be expressed as:

$$
\begin{aligned}
& \iint_{T} \dot{\vartheta}_{R_{2}} \kappa q_{n}(t, \mathbf{x}) \mathrm{d} A \mathrm{~d} t= \\
& \iint_{T} \dot{f}(t, \mathbf{x}) \kappa \lambda \nabla^{\mathrm{T}} \vartheta \mathbf{n} \mathrm{d} A \mathrm{~d} t- \\
& \iint_{T} \dot{\vartheta}_{2} \kappa q_{n}(t, \mathbf{x}) \mathrm{d} A \mathrm{~d} t
\end{aligned}
$$

where: $\mathbf{x}=\left(x_{1}, x_{2}, \ldots, x_{n}\right)^{\mathrm{T}}$ - vector containing the space coordinates for $\dot{f}$ and $q_{n}$.

The next step contains the elimination of the time gradient in the second integral term of Eq. (12). With the relationship

$$
\nabla^{\mathrm{T}} \dot{\vartheta} \nabla \vartheta=\frac{1}{2} \nabla^{\mathrm{T}} \dot{\vartheta} \nabla \vartheta+\frac{1}{2} \nabla^{\mathrm{T}} \vartheta \nabla \dot{\vartheta}=\frac{1}{2}\left(\nabla^{\mathrm{T}} \vartheta \nabla \vartheta\right)^{\bullet}
$$

follows

$$
\iint_{T} \nabla^{\mathrm{T}} \dot{\vartheta} \kappa \lambda \nabla \vartheta \mathrm{d} V \mathrm{~d} t=\frac{1}{2} \int_{T} \int_{G} \kappa \lambda\left(\nabla^{\mathrm{T}} \vartheta \nabla \vartheta\right)^{\bullet} \mathrm{d} V \mathrm{~d} t .
$$

Thus, the derivation of the temperature with respect to the time can be annulled:

$$
\begin{aligned}
& \int_{T} \int_{G} \nabla^{\mathrm{T}} \dot{\xi} \lambda \nabla \vartheta \mathrm{d} V \mathrm{~d} t=\frac{1}{2} \int_{G}\left[\kappa \lambda \nabla^{\mathrm{T}} \vartheta \nabla \vartheta\right]_{t=t_{2}} \mathrm{~d} V- \\
& \frac{1}{2} \int_{G}\left[\kappa \lambda \nabla^{\mathrm{T}} \vartheta \nabla \vartheta\right]_{t=t_{1}} \mathrm{~d} V-\frac{1}{2} \int_{T} \int_{G}(\kappa \lambda)^{\bullet} \nabla^{\mathrm{T}} \vartheta \nabla \vartheta \mathrm{d} V \mathrm{~d} t,
\end{aligned}
$$

where:

$$
(\kappa \lambda)^{\bullet}=\left(\frac{\lambda}{c \rho}\right)^{\bullet}=\frac{\dot{\lambda} c \rho-\lambda \dot{c} \rho-\lambda c \dot{\rho}}{(c \rho)^{2}} .
$$

Because of the material parameters are only defined to be temperature dependent, die partial derivations with respect to the time in Eq. (24) cannot be taken easily. Again, a simple transformation can help solving this problem. As in Eq. (14), it is exemplarily shown on the specific heat capacity $c$, but can be applied to all material properties:

$$
\dot{c}=c^{\prime} \dot{\vartheta}
$$

Thus, Eq. (24) can be expressed as

$$
(\kappa \lambda)^{\bullet}=a^{\prime} \dot{\vartheta}
$$

where:

$$
a^{\prime}=\frac{\lambda^{\prime} c \rho-\lambda c^{\prime} \rho-\lambda c \rho^{\prime}}{(c \rho)^{2}} .
$$

The third term that describes the heat conduction in the functional Eq. (10) will be transformed. By using

$$
\begin{aligned}
& \nabla^{\mathrm{T}}[\lambda \nabla \vartheta]=\nabla^{\mathrm{T}} \lambda \nabla \vartheta+\lambda \nabla^{\mathrm{T}} \nabla \vartheta= \\
& \lambda^{\prime} \nabla^{\mathrm{T}} \vartheta \nabla \vartheta+\lambda \nabla^{\mathrm{T}} \nabla \vartheta,
\end{aligned}
$$

the integral could be expressed as:

$$
\begin{aligned}
& \frac{1}{2} \int_{T} \int_{G}\left(\kappa \nabla^{\mathrm{T}}[\lambda \nabla \vartheta]\right)^{2} \mathrm{~d} V \mathrm{~d} t= \\
& \frac{1}{2} \int_{T} \kappa_{G} \kappa^{2}\left(\lambda^{\prime}\right)^{2}\left(\nabla^{\mathrm{T}} \vartheta \nabla \vartheta\right)^{2} \mathrm{~d} V \mathrm{~d} t+ \\
& \iint_{T} \kappa^{2} \lambda^{\prime} \lambda \nabla^{\mathrm{T}} \vartheta \nabla \vartheta \nabla^{\mathrm{T}} \nabla \vartheta \mathrm{d} V \mathrm{~d} t+ \\
& \frac{1}{2} \int_{T} \int_{G} \kappa^{2} \lambda^{2}\left(\nabla^{\mathrm{T}} \vartheta \nabla \vartheta\right)^{2} \mathrm{~d} V \mathrm{~d} t .
\end{aligned}
$$

At the end, Eq. (28) can also be used to transform the last integral term of Eq. (10) into

$$
\begin{aligned}
& \int_{T} \int_{G} \vartheta \kappa^{2} W \nabla^{\mathrm{T}}[\lambda \nabla \vartheta] \mathrm{d} V \mathrm{~d} t= \\
& \int_{T} \int_{G} \kappa^{2} \lambda^{\prime} W \nabla^{\mathrm{T}} \vartheta \nabla \vartheta \mathrm{d} V \mathrm{~d} t+ \\
& \int_{T} \int_{G} \kappa^{2} \lambda W \nabla^{\mathrm{T}} \nabla \vartheta \mathrm{d} V \mathrm{~d} t .
\end{aligned}
$$


Thus, the functional Eq. (10) for calculating the temperature field can be written in its final form for temperature dependent material properties:

$$
\begin{aligned}
& \Phi=\frac{1}{2} \iint_{T G} \dot{\vartheta}^{2} \mathrm{~d} V \mathrm{~d} t-\int_{T} \int_{R_{1}} \kappa \lambda \dot{f}(t, \mathbf{x}) \nabla^{\mathrm{T}} \vartheta \mathbf{n} \mathrm{d} A \mathrm{~d} t- \\
& \int_{T} \int_{R_{2}} \kappa q_{n}(t, \mathbf{x}) \dot{\vartheta} \mathrm{d} A \mathrm{~d} t+\frac{1}{2} \iint_{G}\left[\kappa \lambda \nabla^{\mathrm{T}} \vartheta \nabla \vartheta\right]_{t=t_{2}} \mathrm{~d} V- \\
& \frac{1}{2} \int_{G}\left[\kappa \lambda \nabla^{\mathrm{T}} \vartheta \nabla \vartheta\right]_{t=t_{1}} \mathrm{~d} V-\frac{1}{2} \iint_{T G} a^{\prime} \dot{\vartheta} \nabla^{\mathrm{T}} \vartheta \nabla \vartheta \mathrm{d} V \mathrm{~d} t+ \\
& \int_{T} \int_{G} \kappa^{\prime} \lambda \dot{\vartheta} \nabla^{\mathrm{T}} \vartheta \nabla \vartheta \mathrm{d} V \mathrm{~d} t+\frac{1}{2} \iint_{T G} \kappa^{2}\left(\lambda^{\prime}\right)^{2}\left(\nabla^{\mathrm{T}} \vartheta \nabla \vartheta\right)^{2} \mathrm{~d} V \mathrm{~d} t+ \\
& \int_{T} \int_{G} \kappa^{2} \lambda^{\prime} \lambda \nabla^{\mathrm{T}} \vartheta \nabla \vartheta \nabla^{\mathrm{T}} \nabla \vartheta \mathrm{d} V \mathrm{~d} t+ \\
& \frac{1}{2} \int_{T} \int_{G} \kappa^{2} \lambda^{2}\left(\nabla^{\mathrm{T}} \vartheta \nabla \vartheta\right)^{2} \mathrm{~d} V \mathrm{~d} t-\iint_{T G} \kappa W \dot{\vartheta} \mathrm{d} V \mathrm{~d} t+ \\
& \iint_{T} \kappa^{2} \lambda^{\prime} W \nabla^{\mathrm{T}} \vartheta \nabla \vartheta \mathrm{d} V \mathrm{~d} t+\iint_{T G} \kappa^{2} \lambda W \nabla^{\mathrm{T}} \nabla \vartheta \mathrm{d} V \mathrm{~d} t,
\end{aligned}
$$

where: term 1 of Eq. (31) - term 1 of Eq. (10); terms 2 to $7(31)$ - term 2(10); terms 8 to $10(31)$ - term $3(10)$; term 11(31) - term 4(10); terms 12 and 13(31) term 5(10).

For some cases, especially when the changes of the temperature field are comparatively small, the assumption of constant material parameters could be valid. Thus, the following statements can be made:

$$
\begin{gathered}
\lambda(\vartheta), c(\vartheta), \rho(\vartheta)=\lambda, c, \rho=\text { const. } \\
\lambda^{\prime}, c^{\prime}, \rho^{\prime}=0 .
\end{gathered}
$$

If the derivations of the material parameters with respect to the temperature are equal to zero, 5 of the 13 integral terms of Eq. (31) can be eliminated and the functional can be expressed in the form derived in (Kaempf 1983):

$$
\begin{aligned}
& \Phi=\frac{1}{2} \iint_{T G} \dot{\vartheta}^{2} \mathrm{~d} V \mathrm{~d} t-\iint_{T} \kappa \lambda \dot{f}(t, \mathbf{x}) \nabla^{\mathrm{T}} \vartheta \mathbf{n} \mathrm{d} A \mathrm{~d} t+ \\
& \int_{T} \int_{R_{2}} \kappa q_{n}(t, \mathbf{x}) \dot{\vartheta} \mathrm{d} A \mathrm{~d} t+\frac{1}{2} \int_{G}\left[\kappa \lambda \nabla^{\mathrm{T}} \vartheta \nabla \vartheta\right]_{t=t_{2}} \mathrm{~d} V- \\
& \frac{1}{2} \int_{G}\left[\kappa \lambda \nabla^{\mathrm{T}} \vartheta \nabla \vartheta\right]_{t=t_{1}} \mathrm{~d} V+\frac{1}{2} \iint_{T G} \kappa^{2} \lambda^{2}\left(\nabla^{\mathrm{T}} \nabla \vartheta\right)^{2} \mathrm{~d} V \mathrm{~d} t- \\
& \int_{T} \int_{G} \kappa W \dot{\vartheta} \mathrm{d} V \mathrm{~d} t+\iint_{T G} \kappa^{2} \lambda W \nabla^{\mathrm{T}} \nabla \vartheta \mathrm{d} V \mathrm{~d} t .
\end{aligned}
$$

For the evaluation of the energy functional (31) and (34), the temperature field at the time $t=t_{1}$ has to be known. For the calculation of stationary temperature fields, the upper limit of the time interval has to be set very high $\left(t_{2} \rightarrow \infty\right)$. However, the elimination of all derivations with respect to the time is not possible, because of the boundary conditions couldn't be evaluated any longer.

For using first type boundary conditions, it has to be ensured, that $f\left(t=t_{1}, \mathbf{x}\right)$ is equal to the temperature at the edge $R_{1}$, because of only the time gradient of $f$ is considered in the functional term. To avoid the modification of the temperature field at the edge for $t=t_{1}$, the constraint temperature at the edge may could be expressed as a function, that increases continuously up to the wanted temperature level.

The introduced functional $\Phi$ describes the total thermal energy of the domain $G$ and within the time interval $T$. The partial differential equation of heat conduction (1) is solved for a temperature field at $t=t_{2}$, that leads to a minimum of the functional value $\Phi$. An advantage of the heat conduction problem is its strict convexity. Thus, there always will be only one temperature field, which satisfies this condition. Because of the boundary conditions are considered with terms within the energy functional, in general the problem is describable as an unconstrained optimization task:

$$
\Phi(\vartheta) \rightarrow \min
$$

\subsection{Numerical implementation}

The introduced methods are numerically implemented to perform $2 \mathrm{D}$ thermal and mechanical analysis on composite cross sections. A 3D implementation could be realized analogously. Cross sections will be divided into parts as shown in Figure 3 (left) for a composite $\mathrm{T}$ cross section. On the one hand side, this allows a different mesh density in different parts of the cross section (e.g. for thin webs of steel beams), on the other hand side this simplifies the evaluation of the energy functional, if every part only consists of one homogenous material. Thus, only at the part transitions inside the cross section, a more accurate treatment has to be made, that considers the materials of the adjoining parts. Every single part is again divided into a grid with equal grid distance, as shown in Figure 3 (right).

For this first implementation, difference quotients are used to approximate the single and double derivation of the temperature field with respect to the coordinate directions. The occurring approximation error decreases with an increasing mesh density. At the edges between two parts, the temperature and the flux density are equal by definition. Considering the edge of part 1, which adjoins to an edge of part 2 with different material, this leads to the following relationships: 

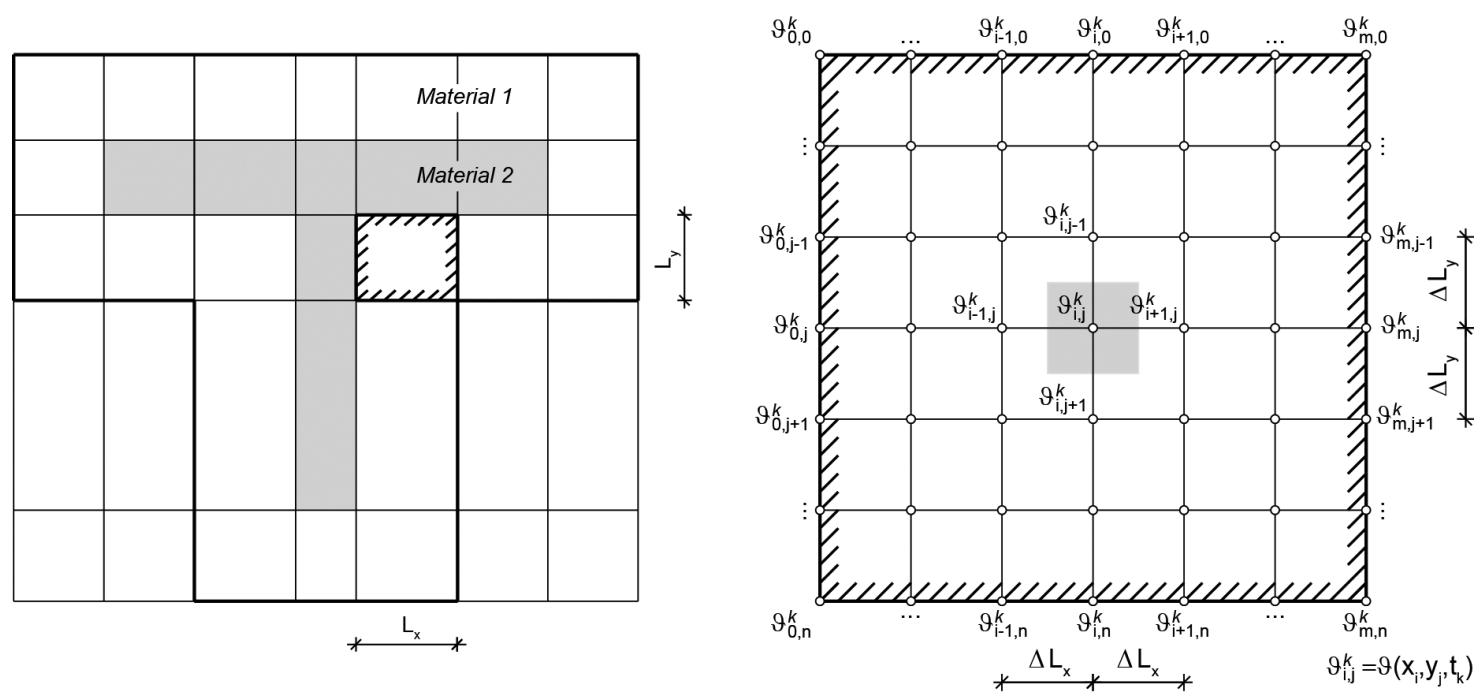

Fig. 3. Division of a composite T cross section into parts (left) and definition of the grid points inside of one part (right)

$$
\begin{gathered}
\vartheta_{1}=\vartheta_{2} ; \\
\frac{\partial \vartheta_{1}}{\partial \mathbf{n}}=\frac{\partial \vartheta_{2}}{\partial \mathbf{n}} \frac{\lambda_{2}}{\lambda_{1}} .
\end{gathered}
$$

For evaluation by using the boundary integrals in Eq. (31) or (34), these conditions can be transformed into:

$$
\begin{gathered}
\dot{f}=\dot{\vartheta}_{1}=\dot{\vartheta}_{2} ; \\
q_{n}=\frac{\left(\frac{\partial \vartheta_{1}}{\partial \mathbf{n}}-\frac{\partial \vartheta_{2}}{\partial \mathbf{n}}\right) \lambda_{1} \lambda_{2}}{\lambda_{2}-\lambda_{1}} .
\end{gathered}
$$

Because of the approximation error within the first derivation of the temperature field in the edge points by using difference quotients, only the continuity of the edge temperatures is ensured in this first approach of the present article. This leads to sufficiently accurate results. The inclusion of shape functions for differentiation, which use the temperature and the temperature gradients of the grid points, would lead to a higher approximation accuracy and enable the easier evaluation of the equations introduced above.

\section{Mechanical analysis of composite cross sections}

\subsection{The energy functional of the mechanical problem}

The mechanical analysis is based on the principle of the minimum of the total potential energy by Lagrange and uses a kinematical description of the mechanical problem. The calculation scheme is shown in Figure 4. Based on a provided compatibility, which should be Bernoulli's hypothesis in this case, the total strain in every fibre $i$ inside a cross section under interaction of normal force and bending moments could be determined by using three parameters, the strain in the coordinate origin $\varepsilon_{0}$ as well as the curvatures $\kappa_{y}$ and $\kappa_{z}$ which describe the gradient of the strain plane (Raue 2007):

$$
\varepsilon_{i, t o t}\left(\varepsilon_{0}, \kappa_{y}, \kappa_{z}\right)=\varepsilon_{0}+\kappa_{y} y_{i}+\kappa_{z} z_{i} .
$$

The stress generating strains $\varepsilon_{\sigma}$ are consisting of the total strains and a share of pre-strains $\varepsilon^{(0)}$, which may are impressed into the cross section from a former load step. For the case of pure mechanical load influences, $\varepsilon^{(0)}$ is equal to zero and the stress generating strains are equal to the total strains.

$$
\varepsilon_{i, \sigma}\left(\varepsilon_{0}, \kappa_{y}, \kappa_{z}\right)=\varepsilon_{i, t o t}\left(\varepsilon_{0}, \kappa_{y}, \kappa_{z}\right)+\varepsilon_{i}^{(0)}
$$

Using the material law, the stress in every fibre can be calculated as well as the specific strain energy $W$, which is defined as the area under the stress-strainfunction (Fig. 4 bottom):

$$
\begin{gathered}
\sigma_{i}\left(\varepsilon_{0}, \kappa_{y}, \kappa_{z}\right)=\sigma\left(\varepsilon_{i, \sigma}\right) ; \\
W_{i}\left(\varepsilon_{0}, \kappa_{y}, \kappa_{z}\right)=\int_{0}^{\varepsilon_{i, \sigma}} \sigma\left(\varepsilon_{\sigma}\right) \mathrm{d} \varepsilon .
\end{gathered}
$$

An advantage of this approach is the possibility of the implementation of arbitrary material models without the application of changes in the calculation scheme, as long as they could be integrated steadily within defined strain ranges. Thus, a physically nonlinear calculation can be done very easily.

The integration of the specific strain energy over the cross section area results in the internal potential energy of the cross section

$$
\Pi_{\text {int }}^{C S}=\Pi_{\text {int }}^{C S}\left(\varepsilon_{0}, \kappa_{y}, \kappa_{z}\right)=\int_{C S} W\left(\varepsilon_{0}, \kappa_{y}, \kappa_{z}\right) \mathrm{d} A .
$$




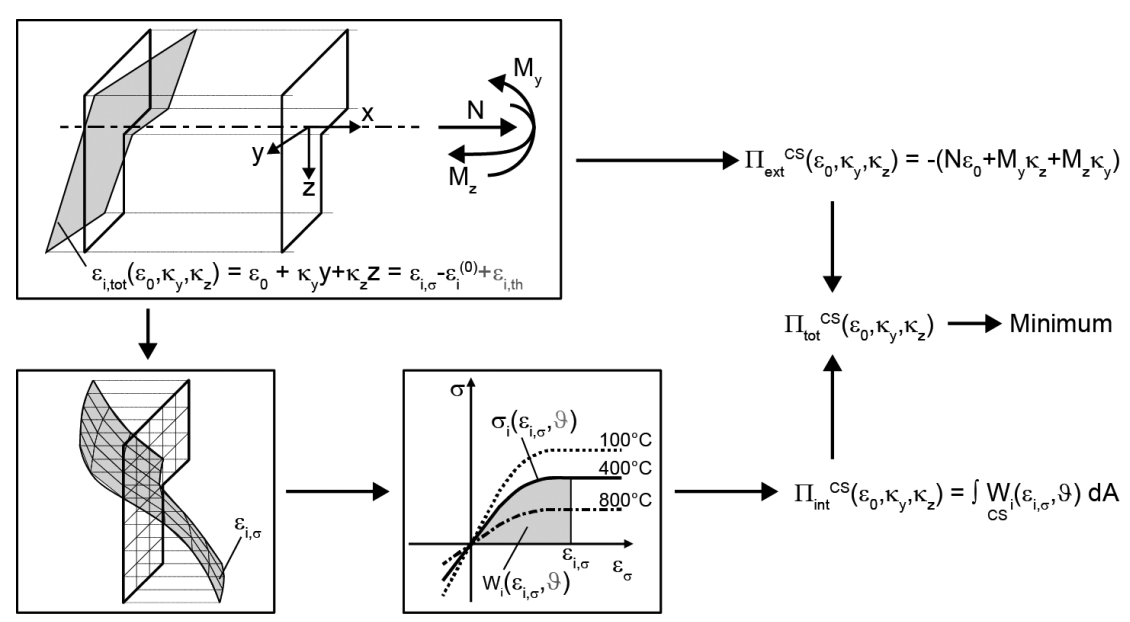

Fig. 4. Calculation scheme for the mechanical analysis based on the minimum of the total potential energy

The potential energy of the external loads is defined by:

$\Pi_{\text {ext }}^{C S}=\Pi_{\text {ext }}^{C S}\left(\varepsilon_{0}, \kappa_{y}, \kappa_{z}\right)=-\left(N \varepsilon_{0}+M_{y} \kappa_{z}+M_{z} \kappa_{y}\right)$.

Summing up the two shares leads to the total potential energy of the cross section, which represents the energy functional of the mechanical problem and which still depends only on the three introduced parameters $\varepsilon_{0}$, $\kappa_{y}$ and $\kappa_{z}$ :

$$
\begin{aligned}
& \Pi_{\text {tot }}^{C S}=\Pi_{\text {tot }}^{C S}\left(\varepsilon_{0}, \kappa_{y}, \kappa_{z}\right)=\Pi_{\text {int }}^{C S}\left(\varepsilon_{0}, \kappa_{y}, \kappa_{z}\right)+ \\
& \Pi_{\text {ext }}^{C S}\left(\varepsilon_{0}, \kappa_{y}, \kappa_{z}\right) .
\end{aligned}
$$

\subsection{Formulation of the optimization task}

Considering the cross section geometry, the material law and the external loads to be known, $\varepsilon_{0}, \kappa_{y}$ and $\kappa_{z}$ are the parameters of the naturally unconstraint optimization task to solve the extremum problem. Of all kinematic possible total strain states inside the cross section, only the one will occur, which leads to a minimum of the total potential:

$$
\Pi_{\text {tot }}^{C S}\left(\varepsilon_{0}, \kappa_{y}, \kappa_{z}\right) \rightarrow \min .
$$

If the task should be restricted, e. g. by prescribing one of the three input parameters to a certain value or by setting limits for calculated strains or stresses, constraints have to be defined. Because of the external potential is equal to zero in the case of no external loads, the total potential is equal to the strain energy of the cross section, as shown in Eq. (48). In this case, the resulting strain state causes a residual stress distribution inside the cross section, which does not lead to internal forces (Schröter 2014).

$$
\Pi_{\text {tot }}^{C S}\left(\varepsilon_{0}, \kappa_{y}, \kappa_{z}\right)=\Pi_{\text {int }}^{C S}\left(\varepsilon_{0}, \kappa_{y}, \kappa_{z}\right) \rightarrow \min .
$$

For both linear and nonlinear material models, the optimization problem behaves strictly convex, as long as the stress-strain-functions are extremely monotonic increasing. In the case of softening or ideal plastic material behaviour, local minima are possible. This phenomenon can be simulated with path-controlled calculations only and needs a stepwise increase of the mechanical exposures and an adaption of the unknown start values for the next calculation step.

\subsection{Equations of equilibrium and internal forces}

The equilibrium conditions are satisfied automatically if the optimization task is solved. Thus, they are not needed for the calculation of the strain and stress distribution inside the cross section. This offers the possibility to use the equations of equilibrium to check the quality of the determined results by comparing the internal forces with the external loads. The internal forces can be calculated with:

$$
\begin{gathered}
N=\int_{C S} \sigma \mathrm{d} A ; \\
M_{y}=\int_{C S} \sigma z \mathrm{~d} A ; \\
M_{z}=\int_{C S} \sigma y \mathrm{~d} A .
\end{gathered}
$$




\subsection{Coupling of the mechanical with the thermal analysis}

As shown in Figure 4, the total strains inside the cross section can be divided into stress generating strains and tension free thermal strains. Consequently, the stress generating strains can be calculated by:

$$
\varepsilon_{i, \sigma}\left(\varepsilon_{0}, \kappa_{y}, \kappa_{z}\right)=\varepsilon_{i, t o t}\left(\varepsilon_{0}, \kappa_{y}, \kappa_{z}\right)-\varepsilon_{i, t h}+\varepsilon_{i}^{(0)} \text {, }
$$

where the thermal strains are determined on the base of the given temperature field:

$$
\varepsilon_{i, t h}=\varepsilon_{i, t h}(\vartheta) .
$$

Using a material law, which may could be time and/or temperature dependent, leads to the stress:

$$
\sigma_{i}\left(\varepsilon_{0}, \kappa_{y}, \kappa_{z}\right)=\sigma\left(\varepsilon_{i, \sigma}, \vartheta, t\right) \text {. }
$$

The specific strain energy can be determined and integrated over the cross section by using Eqs. (43) and (44). The determination of the external and total potential energy remains the same, too. Thus, the optimization task can be established as described in section 2.2.

\section{Application on a principle example}

The performance of the alternative approach for the determination of temperature fields and residual strain and stress states in cross sections exposed to thermal and mechanical influences will be demonstrated on a brief example of a composite cross section subjected to fire conditions. The geometry and material parameters are shown in Figure 5. Because of this cross section is assumed to be a single part of a whole throughed suspended floor, the left and right edge both are modelled as adiabatic edges for the thermal analysis. Only the bottom of the floor is exposed to fire conditions. Further, the reinforcement is neglected during the thermal analysis, because of its influence on the temperature field can be assumed to be comparatively small (Fig. 5, left). For the mechanical analysis, the reinforcement has to be considered, of course. (Fig. 5, right). Therefore, it is assigned to have the same temperature as the surrounding concrete.

The "standard fire curve" taken from (EC1 2002) and shown in Figure 6 (left) describes the time-development of the gas temperature for fire design of structural members. The thermal and mechanical parameters as well as the equations for temperature expansion of the building materials concrete, reinforcement and construction steel are taken from the respective parts of the Eurocode (EC2 2004; EC3 2005). As an example, the temperature dependent developments of the concrete material parameters are shown in Figure 6 (middle and right).

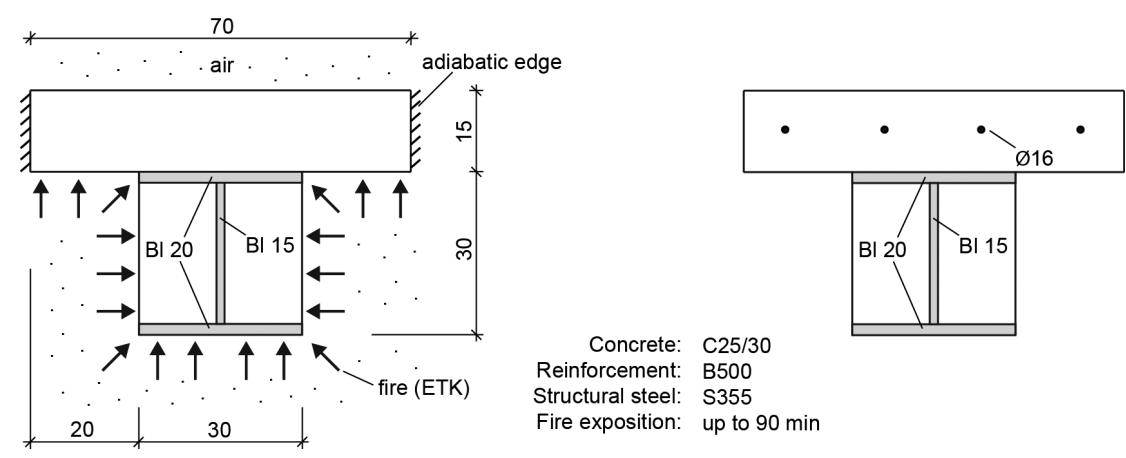

Fig. 5. Cross section properties for the thermal (left) and the mechanical (right) analysis
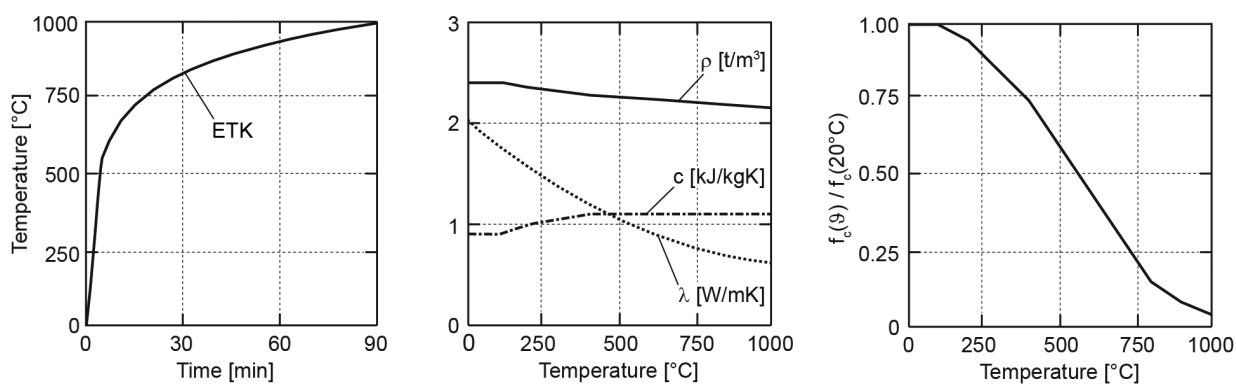

Fig. 6. Development of the outside temperature level (left), temperature dependent thermal material parameters (middle) and mechanical material parameters of concrete (right) 
Using equations for heat convection and radiation, a flux density can be determined and set on the cross section edge (EC1 2002):

$$
q_{n}=\Phi \varepsilon_{m} \varepsilon_{f} \sigma\left[\left(\vartheta_{g a s}+273\right)^{4}-\left(\vartheta_{\text {surf }}+273\right)^{4}\right] \text {, }
$$

where: $\vartheta_{\text {gas }}$ - gas temperature; $\vartheta_{\text {surf }}$ surface temperature, $\Phi$ - configuration factor; $\varepsilon_{\mathrm{m}}$ - emissivity of the surface; $\varepsilon_{\mathrm{f}}$ - emissivity of the radiator $(=1,0$ for fire).

The results of the thermal analysis are shown in Figure 7 for 30, 60 and 90 minutes fire exposition. The influence of the structural steel profile on the temperature field distribution is clearly apparent. Its upper flange and web are representing thermal bridges which conduct a high amount of heat into the inner of the cross section. However, the upper edge of the cross section is nearly untouched due to the fire exposition. The reinforcement was not considered during the thermal analysis, but implemented now for the calculation of the thermal strains as shown in Figure 8 (top left) for $t=60$ minutes. These thermal strains are assumed to be tension free. To satisfy the compatibility (plane cross section), the stress generating strains computed in the mechanical analysis have to be distributed like shown in Figure 8 (bottom left). The corresponding residual stresses (Fig. 8 bottom right) show, that the edges of the cross section, which are heated the most, underwent compression, whilst the shielded inner parts are under tension.

\section{Conclusions}

The introduced numerical approach is a powerful method for the coupled thermal and mechanical analysis of composite cross sections subjected to combined thermal and mechanical influences, which uses the mathematical optimization as a uniform methodical base.
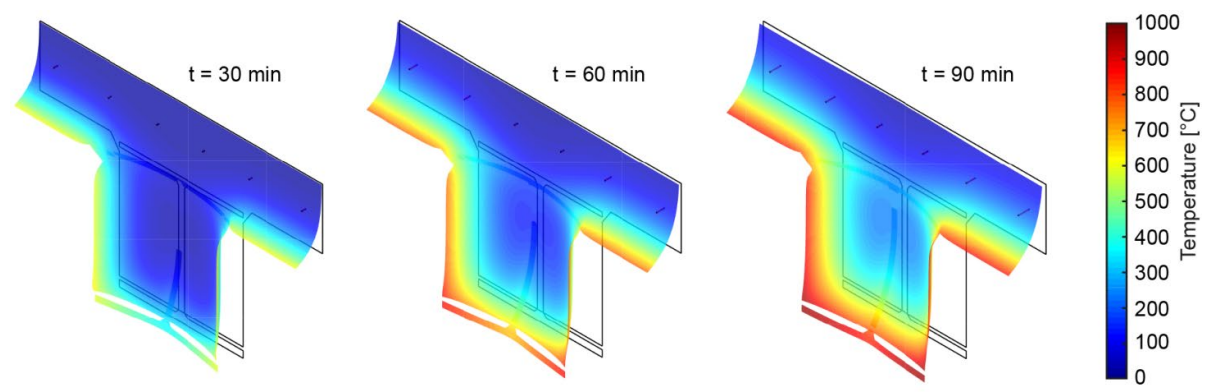

Fig. 7. Temperature fields for 30 (left), 60 (middle) and 90 minutes fire exposition (right)
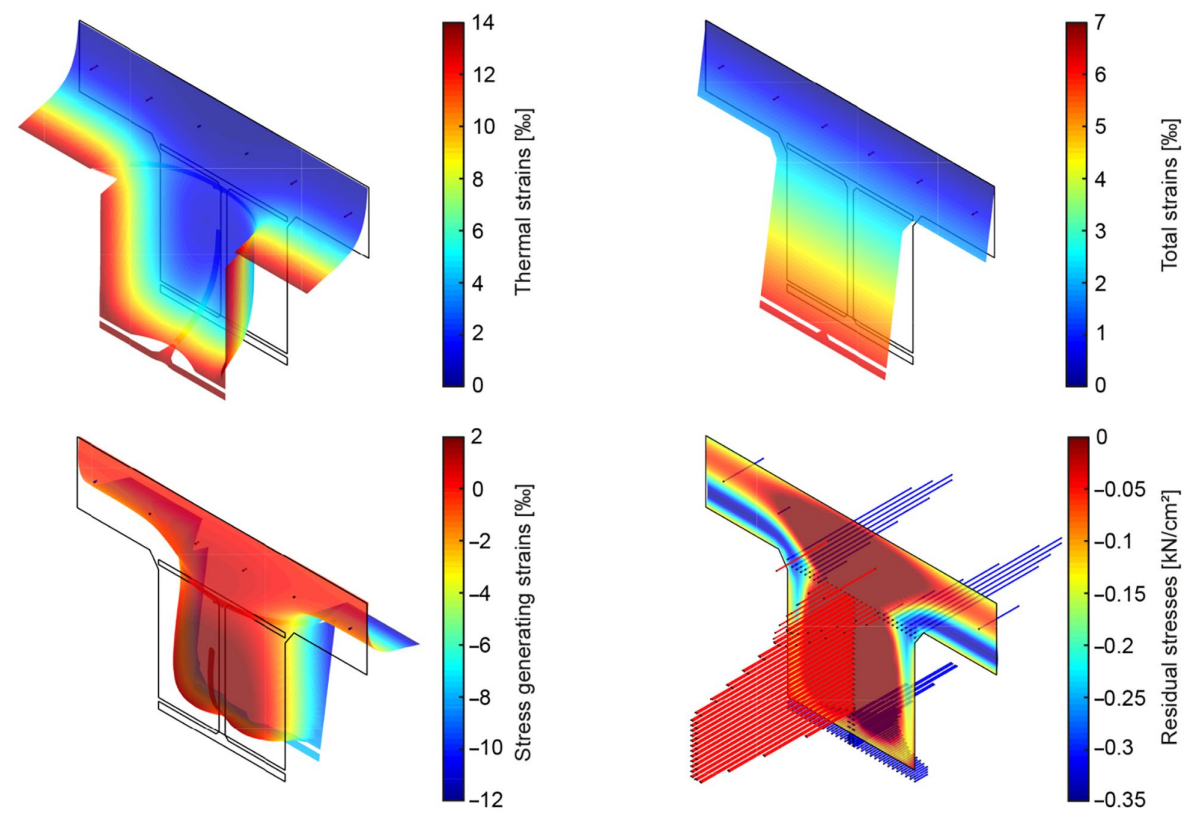

Fig. 8. Thermal, stress free strains (top left), stress generating strains (bottom left), total strains (top right) and residual stresses (bottom right) for 60 minutes fire exposition 
The derived optimization task for the heat conduction problem represents an implicit method, whose stability does not impose any stability criteria in time or space discretisation. Hence, the maximum time step is only limited by the required accuracy. Furthermore, higher order shape functions can help to increase the accuracy in the approximation of the temperature field and its gradients and to satisfy the compatibility at material transitions.

The coupling between the thermal and mechanical analysis is performed by using the thermal (stress free) strains and temperature dependent material parameters. Through the consideration of the compatibility conditions (e. g. plane cross section after deformation), the stress generating as well as the total strains can be determined by solving the optimization task of the mechanical problem. The residual strains and stresses can be superimposed with arbitrary mechanical loads or constraints.

An advantage of the presented methods is the ability of easy implementation of the algorithms considering nonlinear material models and parameters. The performance is demonstrated on a composite cross section subjected to fire conditions. By modification of the thermal and/or mechanical influences as well as the material laws, other phenomena, like solar radiation, technical heating and cooling or the hydration period of young concrete can be simulated. In the case of young concrete, also the mechanical analysis has to be performed in time increments, because of the time dependence of the mechanical material parameters.

The explained methods can be extended from the cross section level to the element level by the application of pseudo-3D or 3D temperature field analysis, by the use of equations for the compatibility between the element deformations and the cross section distortions and by the integration of the strain energy over the element using quadrature formulas. Thus, the geometrically and physically nonlinear analysis of whole structures subjected to combined mechanical and thermal constraints becomes possible.

\section{Disclosure statement}

The present article is authored without any competing financial, professional or personal interests from other parties.

\section{References}

Achenbach, M.; Morgenthal, G. 2014. Vollprobabilistische Analyse von Stahlbetonwänden unter Brandeinwirkung, Bauingenieur 89(11): 478-486.

Baehr, H. D.; Stephan, K. 2013. Wärme- und Stoffübertragung. Berlin, Heidelberg: Springer.

https://doi.org/10.1007/978-3-642-36558-4

EC1 2002. EN 1991 Eurocode 1: Actions on structures - Part 1-2: General actions - Actions on structures exposed to fire.

EC2 2004. EN 1992 Eurocode 2: Design of concrete structures Part 1-2: General rules - Structural fire design.

EC3 2005. EN 1993 Eurocode 3: Design of steel structures - Part 1-2: General rules - Structural fire design.

Häfner, F.; Sames, D.; Voigt, H.-D. 1992. Wärme- und Stofftransport: Mathematische Methoden. Berlin, Heidelberg: Springer. https://doi.org/10.1007/978-3-662-00982-6

Jung, B.; Morgenthal, G.; Xu, D. 2013. Integral bridges: sensitivity of limit state modelling, Bautechnik Sonderdruck "Modellqualitäten" 90: 32-40.

Kaempf, B. 1983. Ein Extremal-Variationsprinzip für die instationäre Wärmeleitung mit einer Anwendung auf thermoelastische Probleme unter Verwendung der finiten Elemente, Mitteilungen aus dem Institut für Mechanik Nr. 35. Bochum.

Mazilu, P. 1982. Das Variationsprinzip der virtuellen Energieübertragung, Mitteilungen aus dem Institut für Mechanik, Nr. 33. Bochum.

Morgenthal, G.; Olney, P. 2016. Concrete Hinges and Integral Bridge Piers, Journal of Bridge Engineering 21(1): 060150051-060150056.

https://doi.org/10.1061/(ASCE)BE.1943-5592.0000783

Raue, E. 2005. Nichtlineare Querschnittsanalyse als Optimierungsproblem, Bautechnik 82(11): 796-809.

https://doi.org/10.1002/bate.200590232

Raue, E. 2007. Non-linear analysis of composite cross-sections by non-linear optimization, in Proceedings of the $9^{\text {th }}$ International Conference "Modern Building Materials, Structures and Techniques", 16-18 May 2007, Vilnius, Lithuania.

Schröter, H. 2014. Nichtlineare Analyse von Verbundelementen auf der Grundlage von Energieprinzipien unter Anwendung der mathematischen Optimierung: Dissertation. Weimar. 
Christopher TAUBE. PhD student and research associate at the Department of Modelling and Simulation of Structures at BauhausUniversity Weimar. Research interests in physically and geometrically nonlinear analysis of reinforced concrete and composite structures, simulation of temperature effects due to fire exposition and hydration of young concrete, and application of alternative approaches for numerical analyses in structural engineering.

Hans-Georg TIMMLER. PhD graduate, research associate and lecturer at the Department of Modelling and Simulation of Structures at Bauhaus-University Weimar. Research topics are physically and geometrically nonlinear analysis of reinforced concrete and composite structures, experimental and numerical investigations on short- and long-time bearing behaviour of concrete members, shakedown analysis and advanced capacity design of cyclic loaded structures.

Marcel HELMRICH. Civil engineering student at Bauhaus-University Weimar. Student assistant at the Department of Modelling and Simulation of Structures. Interested in temperature field analysis using conventional and alternative numerical approaches as well as thermal material properties of fire exposed and young concrete.

Guido MORGENTHAL. Diploma in Structural Engineering from TU Berlin, MSc from Imperial College London and MPhil and $\mathrm{PhD}$ degrees from Cambridge University. Consultant engineer on many long-span bridge projects. Since 2010 Professor at Bauhaus University Weimar and Director of its Institute of Structural Engineering. Research interests in wind engineering, numerical methods in structural engineering and fluid dynamics as well as modern monitoring systems in civil engineering. 